\title{
Navigating curriculum transformation: charting our course
}

\author{
Astrid Turner ${ }^{1}$; Irene Lubbe ${ }^{2}$; Liz Wolvaardt ${ }^{1}$; Lizeka Napoles ${ }^{1}$ \\ ${ }^{1}$ The School of Health Systems and Public Health, University of Pretoria, South Africa, \\ ${ }^{2}$ Department for Education Innovation, University of Pretoria, South Africa.
}

\begin{abstract}
The 2015 student-led \#FeesMustFall campaign in South Africa initiated at some universities, and accelerated at others, indepth discussions and reflections about curriculum transformation within the perceived ivory towers of learning. Three years later, the mandate to implement transformation is clear from all levels but what is uncertain is the operationalization of the official transformation framework at the University of Pretoria. The aim of the project presented is to chart the process followed by one of the four Schools of the Faculty of Health Sciences to deconstruct this transformation framework. As part of a three phased programme, a workshop of diverse staff using a modified nominal technique was held in 2018. It resulted in a visual tool of 19 specific statements considered evidence of personal practice that supports and advances the drive for transformation. This tool will be used to share practices and instil individual and School accountability for everone's role in curriculum transformation. In conclusion, complex policy ideals and technical terminology can be translated into practical, appropriate actions by a diverse group of staff and students. The use of a modified nominal group technique was an efficient way to do this by generating an easy-to-use visual tool.
\end{abstract}

Keywords: Curriculum transformation; epistemology; pedagogy; classroom practices; institutional culture; capacity building. 


\section{Introduction}

In mid-October 2015, South Africa was confronted with intense student-led and citizen supported protests against the rising and unaffordable exclusionary fees as well as the lack of sufficient progress towards transformation within its Higher Education Institutions (HEIs). This \#FeesMustFall campaign initiated at some HEIs, and accelerated at others, the discourse around transformation within the perceived ivory towers of learning (SA.DHET, 2017; CHET 2017). Three years later, the mandate to implement curriculum and broader transformation is clear from the Department of Higher Education and Training (Cloete et al, 2017), and society. The mandate has permeated down to our own setting, the School of Health Systems and Public Health (SHSPH) in the Faculty of Health Sciences at the University of Pretoria (UP). What is not clear are the day-to-day actions that all staff need to undertake to contribute towards these transformed spaces.

This paper reports on the the four drivers of the University's curriculum transformation framework and the process followed by the SHSPH to deconstruct, interpret and internalize this official framework. The outcomes of this process - a visual tool - that is contextually appropriate and grounded in our own understanding is presented .

\section{Transformation framework}

In response to the national agenda for curriculum transformation in institutions of Higher Education, the UP developed a framework to drive the agenda within the University. After extensive consultations with both external and internal stakeholders, four curriculum transformation drivers were identified (University of Pretoria, 2017). The intention is to use these transformation drivers as lenses through which our current practices can be interrogated and new approaches shaped.

The four drivers with summarized descriptions include: 1) Responsiveness to social context: a transforming curriculum that responds to local and global contexts, histories, realities and problems; 2) Epistemological diversity: bringing marginalised groups, experiences, knowledges and worldviews emanating from Africa and the Global South to the centre of the curriculum; 3) Renewal of pedagogy and classroom practices: responsiveness to and training in new pedagogical methodologies and approaches within disciplines and finally, 4) An institutional culture of openness and critical reflection: exposing and resisting the subliminal practices of the hidden curriculum that are part of South Africa's legacy of discrimination.

All faculties within the university were tasked to develop plans demonstrating how they would use the identified drivers to transform the various curricula. 
The Faculty of Health Sciences is comprised of four Schools (Dentistry, Healthcare Sciences, Health Systems and Public Health, and Medicine), each of which has a unique history, structure, culture, ethos and vision for the future which influences the way in which the members of each understand, approach and conceptualise their curriculum transformation journey. The Faculty tasked its Teaching and Learning Committee (TLC) with members representing each School to develop specific transformation plans for their programmes and needs. One of the activities in the plan for the SHSPH was to engage academic and support staff in the School, to create awareness and get a 'buy-in' about the four drivers that would guide our curriculum transformation.

\section{Method}

The SHSPH TLC members devised three phases to the programme 1) pre-workshop dissemination and sensitization to the drivers of the official university document with materials posted in the tearoom and circulated electronically two weeks prior to 2) the workshop of academic and administrative staff (to be described in greater detail below) and 3) a post-workshop reflection session of the committee. The entire programme was conducted from December 2017 to March 2018.

The following strategy was used for the workshop. After a brief welcome, each TLC member briefed the participants on the meaning of one driver term. An adapted nominal group technique (NGT) of four mixed groups [academic staff $(n=16)$, senior students $(n=5)$ and administrative $(n=5)$ staff] with an assigned TLC staff member (including the education consultant of the Faculty of Health Sciences) was created to ensure that perspectives from different levels of staff was included and focussed towards the workshop's overall outcome of a core list of practical ways of implementation towards transformation at the SHSPH.

The NGT, as originally developed by Delbecq and vandeVen (1971), is a structured method for brainstorming in group context where there is a diverse group of participants. A NGT eliminates power imbalances (for example, due to role, seniority, or gender) in the room, encouraging the uninhabited sharing of thoughts (Roets and Lubbe, 2015). It further aids in problem identification, generating a variety of solutions as well prioritization and decisionmaking in a group-context thereby giving voice and choice to all participants.

Each group was therefore responsible for a driver term and was provided with flipchart and markers and an envelope of instructions, including a copy of the official transformation document. They were given 15 minutes to compile at least six practical activities relating to their driver term. During the NGT-session, group presentations was done where the flipcharts was displayed, which resulted in the identification of common understandings and a plurality of suggestions. Due to time constraints of academic staff and to ensure that the environment remained focused but lighthearted, the technique was modified where 
informal tallying was done with each person in the room (excluding the TLC members) being given three sticker dots to vote on each driver's activities. Consensus was thus not the aim of the NGT, but rather to generate and prioritise a variety of strategies that can be suggested for implementation depending on the didactic and personal approach of the individual.

The project was concluded a week later with a quality assurance step by the TLC to ensure the items that were voted on were extracted and that the language was clear. Items that were related to the Faculty (rather than the SHSPH) were not included (these strategies were shared with Faculty via the internal structure).

\section{Results}

The study resulted in 19 specific statements considered evidence of personal practice that supports and advances the drive for transformation. A non-specific statement (other) was added to each of the four drivers in order to stimulate innovation and allow for variances in job descriptions (See Figure 1 for an anonymized version of the "star chart").

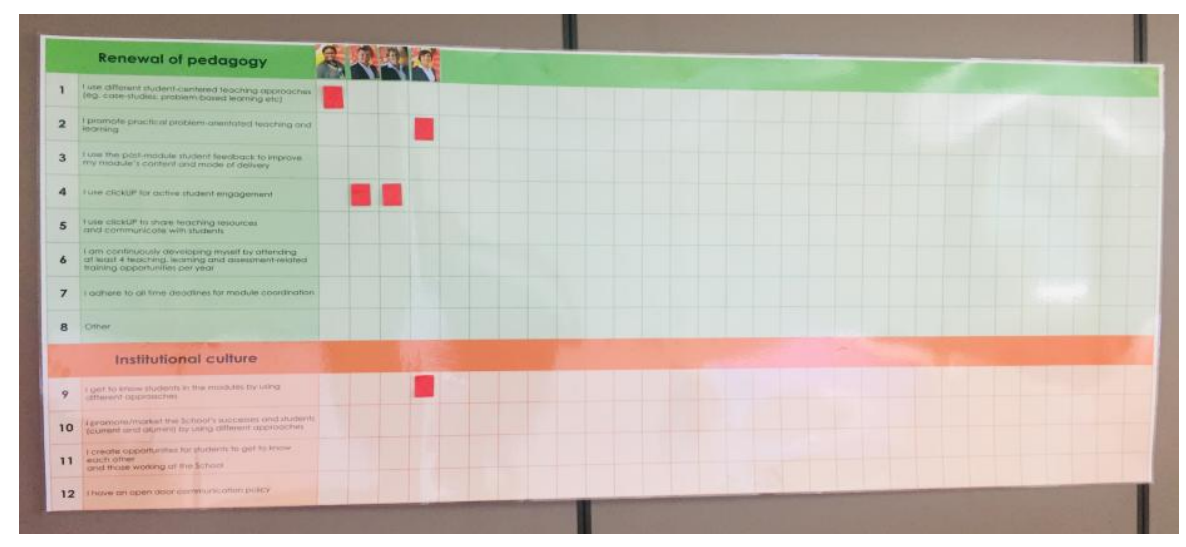

Figure 1. Extract of the star chart of transformation (2019).

The four drivers and the associated statements are:

\section{Responsiveness to social context $(n=3)$}

- I include and encourage sound ethical principles, behaviour and advocacy in my modules and conduct

- I engage in discussion about social issues

- I participate in School and/or Faculty community outreach, transformation and awareness activities 
Astrid Turner; Irene Lubbe; Liz Wolvaardt; Lizeka Napoles 


\section{Epistemological diversity $(n=4)$}

- I use contemporary African examples, content and context in my teaching /facilitation (e.g. readings, case-studies, open education resources [OERs] etc.)

- I contribute to and/or create content related to the Africanisation of the curriculum e.g. by creating and sharing OERs such as YouTube videos and publishing in open-access journals etc.

- I value other knowledge-base systems

- I use and encourage students to use local resources (eg. websites, books etc.)

\section{Renewal of pedagogy $(n=7)$}

- I use different student-centered teaching approaches (eg. case-studies, problembased learning etc.)

- I promote practical problem-orientated teaching and learning

- I use the post-module student feedback to improve my module's content and mode of delivery

- I use clickUP (the university's learning management system) for active student engagement

- I use clickUP to share teaching resources and communicate with students

- I am continuously developing myself by attending at least four teaching, learning and assessment-related training opportunities per year

- I adhere to all time deadlines for module coordination

\section{Institutional culture $(\mathbf{n}=5)$}

- I get to know students in the modules by using different approaches

- I promote/market the School's successes and students (current and alumni) by using different approaches

- I create opportunities for students to get to know each other and those working at the School

- I have an open door communication policy

- I support and/or contribute to School seminars, $\mathrm{PhD}$ presentations etc. 


\section{Discussion and conclusion}

The use of the modified NGT was a simple but effective method to deconstruct the transformation drivers, stimulate discussion about what these drivers mean in practical terms and develop an understanding of both the purpose and practice of these drivers in our context. The activity also resulted in a visual tool and commitment to transformation, against which the academic and administrative staff can be held accountable. The benefits of using this method included team-building and interdisciplinary participation despite the time limitation.

Complex policy ideals and technical terminology can be translated into practical, easy-tounderstand actions by a diverse group of staff and students. The use of a modified NGT was an efficient way to do this and to generate a visual tool that is easy to use. Once field tested, the statements could be considered for formal inclusion in staff's performance agreements. This paper reported on the development of this tool, but what is not yet known is whether the use of this tool will translate into the desired transformation. Measuring the effect of this tool should be the focus of future work.

\section{References}

CHET. 2017. Council On Higher Education Annual Report 2015/16. Online https://www.che.ac.za/sites/default/files/publications/CHE\%20Annual\%20Report\%202 015_16.pdf

Cloete, N., Maassen, P., Fehnel, R., Moja, T., Perold, H. \& Gibbon. T. (2017). Transformation In Higher Education. Global Pressures and Local Realities in South Africa. Department of Higher Education. Kluwer Academic Publishers, The Netherlands

Delbecq, A.L. \& VandeVen, A.H. (1971). A Group Process Model for Problem Identification and Program Planning. Journal of Applied Behavioral Science, 7, 46691. doi:10.1177/002188637100700404

Roets, L. \& Lubbe, J.C. (2015). Power imbalances in research: a step by step illustration of an adapted multiple nominal group analysis. International Journal of Arts \& Sciences, (8)5, 151-164.

SA.DHET. (South Africa, Department of Higher Education and Training). 2017. Commission of Inquiry into Higher Education and Training. Released online http://www.dhet.gov.za/Commissions\%20Reports/Report\%20of\%20Commission\%20of $\% 20$ Inquiry\%20into\%20the\%20Feasibility\%20of\%20Making\%20Higher\%20Educatio n\%20and\%20Training\%20Fee-free\%20in\%20South\%20Africa.pdf

University of Pretoria, Work stream on curriculum transformation (2017). Curriculum Transformation Framework Document: Reimagining curricula for a just university in a vibrant democracy. Retrieved from University of Pretoria: https://www.up.ac.za/faculty-of-law/article/2291240/speakoutup 